\title{
Beam switched antenna using inverted $F$ antenna for mobile terminal application
}

\author{
Bakar Rohani $^{1 \mathrm{a})}$, Shun Yonezawa ${ }^{1 \mathrm{~b})}$, Hiroyuki Arai ${ }^{1}$, \\ Amane Miura ${ }^{2}$, and Hiroyuki Tsuji ${ }^{2}$ \\ ${ }^{1}$ Graduate School of Engineering, Yokohama National University, \\ 79-5 Tokiwadai, Hodogaya-ku, Yokohama 240-8501, Japan \\ ${ }^{2}$ National Institute of Information and Communications Technology (NICT), \\ 4-2-1 Nukui-Kitamachi, Koganei-cho, Tokyo 184-8795, Japan \\ a) rohani-bakar-ng@ynu.ac.jp \\ b) yonezawa-shun-fm@ynu.jp
}

\begin{abstract}
This paper presents a super directive, beam switched antenna for mobile terminal application in satellite communication system. The antenna employs two inverted $\mathrm{F}$ elements mounted on a ground plane which is optimized to be as the same size as typical smartphones. The inverted $\mathrm{F}$ elements are fed alternatingly while the other one acting as parasitic element. A simple feeding circuit utilizing PIN diodes is used to switch the unidirectional radiation pattern in yz-plane into two opposite directions. This paper discusses the complete prototype antenna which is fabricated to demonstrate the switching of the beam patterns in vertical plane.
\end{abstract}

Keywords: beam switched, inverted F, mobile terminal, PIN diode, super directivity array

Classification: Antennas and Propagation

\section{References}

[1] T. Minowa, M. Tanaka, N. Hamamoto, Y. Fujino, N. Nishinaga, R. Miura, and K. Suzuki, "Satellite/terrestrial integrated mobile communication system for nation's security and safety," IEICE Trans., vol. J91-B, no. 12, pp. 1629-1640, 2008.

[2] A. Haskou, A. Sharaiha, and S. Collardey, "Design of small parasitic loaded superdirective end-fire antenna arrays," IEEE Trans. Antennas Propag., vol. 63, no. 12, pp. 5456-5464, Dec. 2015. DOI:10.1109/TAP.2015.2496112

[3] H. Nakamura and H. Arai, "A study of super gain effect of inverted F antenna array” 2014 IEICE Society Conf., pp. B-1-118, Sept. 2014.

\section{Introduction}

The spread of portable terminals for wireless mobile satellite communication entails for miniaturization design with high gain antenna characteristic. Satellite commu- 
nication requires high gain antenna for carrying out the long-distance communication while at the same time capable of suppressing unnecessary radiation that can influence human body. In mobile satellite communication, the link margin in the link budget of voice communication by a portable terminal is quite small with $3.4 \mathrm{~dB}$ for forward link and $1.1 \mathrm{~dB}$ for return link [1]. Hence, the antenna gain is crucial and needs to be enhanced when designing the antenna. Tremendous progress in miniaturizing wireless communication equipment requires power-saving, low profile compact antenna. Furthermore, the exposure of radio waves radiation to human body can be reduced by using unidirectional radiating antenna instead of conventional omni directional antenna. In this paper, we propose a high gain, small antenna satisfying these requirements where a single directivity with switching property is obtained with super directivity effect and small inter-element spacing. A printed half-dipole antenna having super directivity effect is reported in [2] but the structure is quite large because a decent size is needed to cancel the current distribution. In [3], an inverted $\mathrm{F}$ antenna mounted on a broad base plate produces a super gain effect with directivity switching capability. Referring to this technique, this paper proposes a beam switched antenna with more compact and smaller in size using inverted $\mathrm{F}$ antenna capable of switched, unidirectional directional radiation pattern perpendicular to the antenna element. The ground plane which is adjusted to be the size of typical smartphone is utilized effectively to control the current distribution. The effectiveness of the antenna is demonstrated in both simulation and measurement results.

\section{Antenna design}

The proposed antenna is intended for portable terminal of mobile satellite communication operating at frequency $2 \mathrm{GHz}$. The antenna will be fixed on top of the mobile terminal and the objective is to increase the gain of the unidirectional radiation pattern. Basic geometry for the proposed antenna is shown in Fig. 1(a) consisting of two parts, the ground plane (GP) and the antenna pack. Two inverted F elements is placed $0.05 \lambda$ in distance from each other and each element can be excited independently making the other as parasitic element. The excitation produces a unidirectional beam pattern due to the strong coupling between the elements. Capacitance is inserted as a decoupling circuit to improve the isolation between the elements. The impedance is matched at resonance frequency as shown in Sparameter result in Fig. 1(b) where $S_{11}$ of below $-10 \mathrm{~dB}$ is achieved.

The original GP which is just a base plate is improved by adding a side plate and the dimensions are optimized where the impact of the GP is shown in current distribution results as described in Fig. 1(c). Three conditions are investigated where in (a) which the antenna pack is directly connected to the GP, most of the currents flow in the connected part showing that the influence of the GP is large. Therefore, a wire is used as conductor in (b) to reduce the current flowing through the connecting part. In (c), the coupling current flowing from the GP is totally cut off when an inductor is inserted in the conductor offering the optimized structure. The size of the side GP is optimized as the size of the typical smartphone. The directivity of the pattern is shown in Fig. 1(d) where the color of the line 


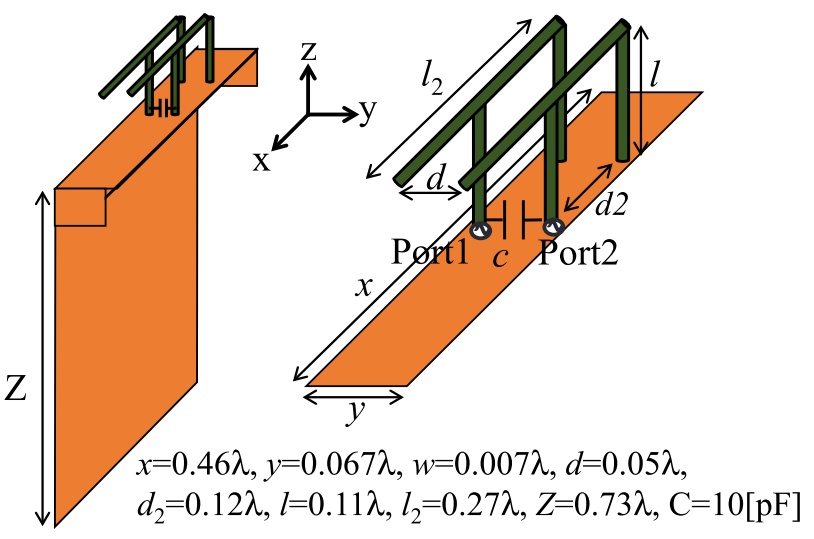

(a)

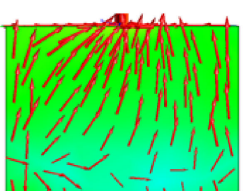

a) not separated

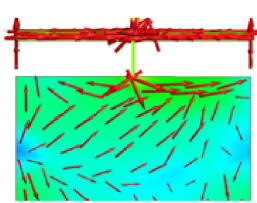

b) add conductor

(c)

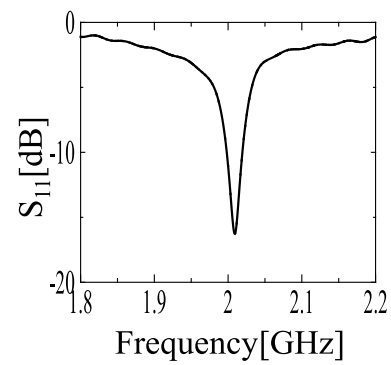

(b)

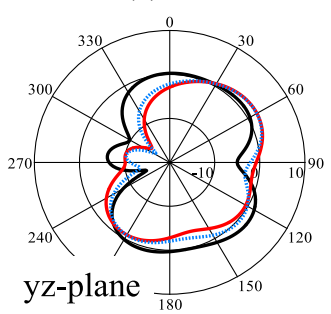

(d)

Fig. 1. (a) Basic geometry of the proposed antenna with (b) $S_{11}$ result. (c) Investigation of GP and (d) correspond radiation patterns.

corresponds to the investigation done in Fig. 1(c). The radiation patterns show improvements in directivity from black line to blue line for added conductor and inductor where the radiation in the opposite direction (at angle $230^{\circ}$ ) is reduced while the radiation at the targeted angle is increased producing a high gain directivity at angle $60^{\circ}$.

The final structure of the proposed antenna is shown in Fig. 2(a) with the optimized dimensions which provide good radiation patterns especially in yz-plane. Fig. 2(b) shows the simulation results for the radiation patterns for Port 1 (black line) and Port 2 (red line) where similar patterns are obtained for both ports and good switching between them are confirmed. The maximum gain obtained at $60^{\circ}$ direction is $3.2 \mathrm{dBi}$ for both ports. A feeding circuit with switching capability is designed where two PIN diodes are used as shown in Fig. 2(c). The PIN diodes can be switched on and off providing one open and one short port thus switching the radiation patterns in two opposite directions. The reflection characteristics are shown in the S-parameter results in Fig. 2(d) which show good isolations $\left(\mathrm{S}_{21}\right.$ and $S_{31}$ ) between the ports are obtained.

\section{Fabrication and experiment results}

The prototype of the proposed antenna together with the feeding circuit is fabricated where the feeding circuit is grounded to the GP. The size of the GP is optimized to the size of a typical smartphone and the fabricated antenna is shown in Fig. 3(a). The S-parameter results show good characteristics where the resonance points for measurement result shift from the simulation results for both ports to be closer to $2 \mathrm{GHz}$. The reflection characteristics are confirmed below $-10 \mathrm{~dB}$ at the design frequency. 
The complete radiation pattern results for both ports in xy-, yz- and zx-plane are shown in Fig. 3(b). Port 1 is described in black line while Port 2 is represented in red line. In general, the measurement results are in agreement with the simulation results which show the reliability of the proposed design. The directivity is confirmed at angle $60^{\circ}$ and the switching also is obtained when the ports are fed

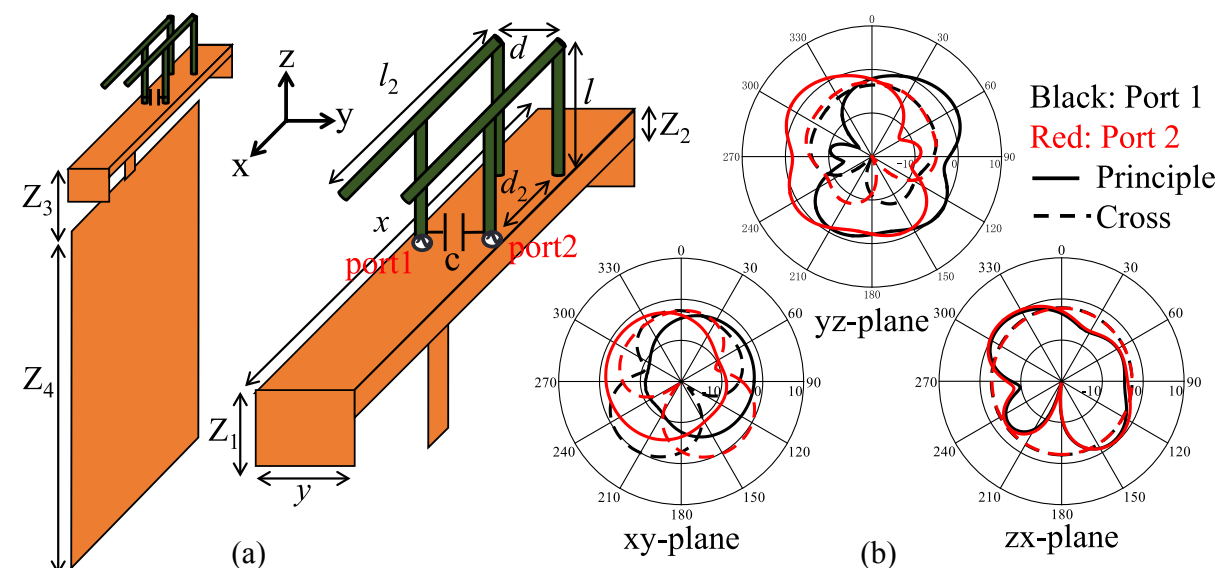

$x=0.46 \lambda, y=0.067 \lambda, w=0.007 \lambda$, $d=0.05 \lambda, d_{2}=0.12 \lambda, l=0.11 \lambda$, $l_{2}=0.27 \lambda, Z=0.067 \lambda, Z_{2}=0.013 \lambda$, $\mathrm{Z}_{3}=0.067 \lambda, \mathrm{Z}=0.733 \lambda, \mathrm{C}=10[\mathrm{pF}]$

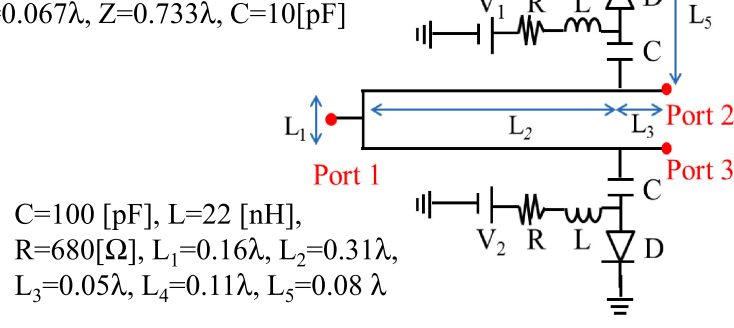

(c)

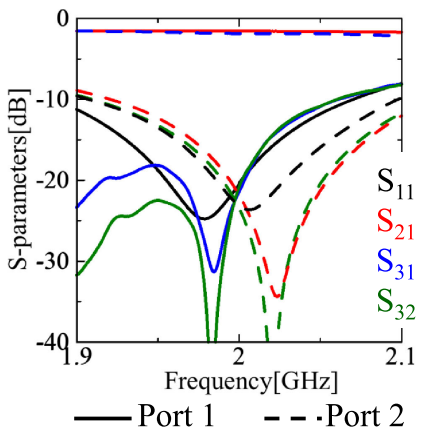

(d)

Fig. 2. (a) Final geometry of the proposed antenna and (b) radiation patterns for Port 1 and 2. (c) The feeding circuit and (d) its reflection characteristics.
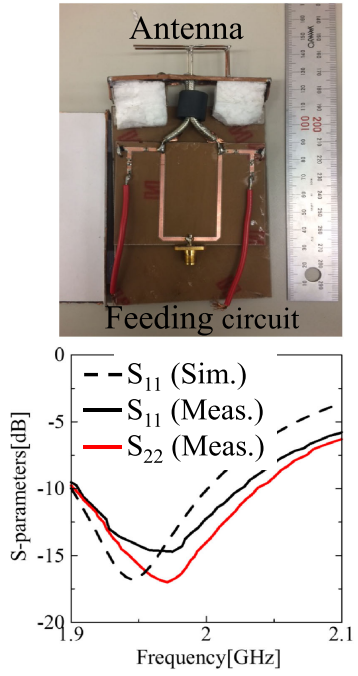

(a)
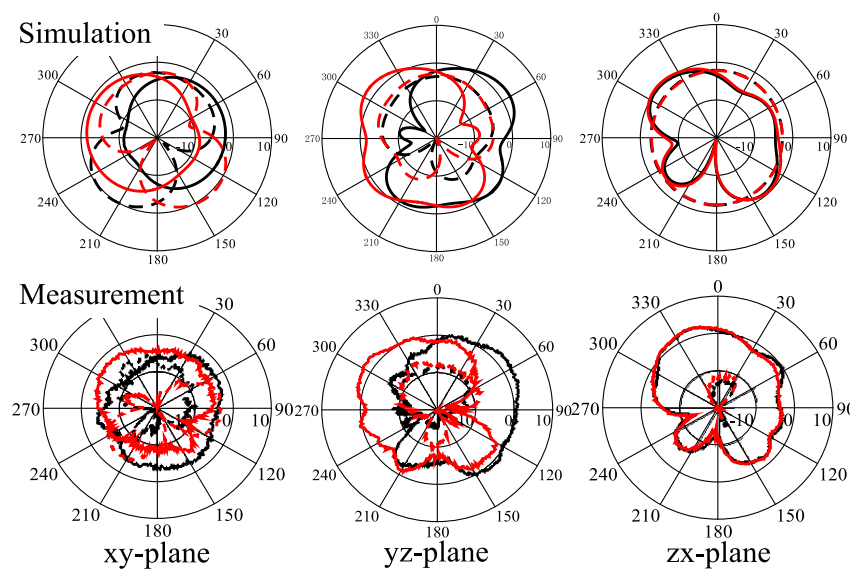

Black: Port 1 Red: Port $2 \longrightarrow$ Principle - - Cross

(b)
๑) IEICE 2017

DOI: 10.1587/comex.2016SPL0016 Received December 7, 2016 Accepted December 27, 2016 Publicized February 21, 2017 Copyedited June 1, 2017 
alternatingly. The maximum gain obtained in simulation and measurement results are $3.2 \mathrm{dBi}$ and $2.5 \mathrm{dBi}$, respectively, with half power beamwidth (HPBW) of approximately $20 \mathrm{MHz}$. In addition, the gain is increased $1 \mathrm{~dB}$ as compared to the inversed $\mathrm{F}$ antenna in the previous research [3].

\section{Conclusion}

This paper proposed a beam switched antenna for mobile terminal application in mobile satellite communications utilizing inverted $\mathrm{F}$ antenna to obtained a super directivity array. A method of cancelling the coupling current distribution flowing from the GP was also proposed by inserting the inductor in the conductor that connecting the GP. Furthermore, a feeding circuit with switching capability was designed employing PIN dioded in the circuit, feeding the port On and OFF alternatingly. The efficiency of the beams switching was confirmed by simulation and measurement results which show an agreement between both results. The antenna has a high gain, unidirectional patterns in yz-plane that can be switched in two directions which favorable for satellite communication applications. 